\title{
A Note on Discrete Multitime Recurrences of Samuelson-Hicks Type
}

\author{
Bruno Antonio Pansera ${ }^{1,2}$ and Francesco Strati ${ }^{1,2,3}$ \\ ${ }^{1}$ Department of Law and Economics, University Mediterranea of Reggio Calabria, Reggio Calabria, Italy \\ ${ }^{2}$ Decisions Lab, University Mediterranea of Reggio Calabria, Reggio Calabria, Italy \\ ${ }^{3}$ Department of Economics and Statistics, University of Siena, Siena, Italy
}

Correspondence should be addressed to Francesco Strati; francesco.strati@unisi.it

Received 11 August 2016; Accepted 5 October 2016

Academic Editor: Gafurjan Ibragimov

Copyright (C) 2016 B. A. Pansera and F. Strati. This is an open access article distributed under the Creative Commons Attribution License, which permits unrestricted use, distribution, and reproduction in any medium, provided the original work is properly cited.

By this work, we aim at fostering further research on the applications of multitime recurrences. In particular, we shall apply this method by generalizing the Samuelson-Hicks model so as to make the new concept of time that this method proposes clear. In particular, the multitime approach decomposes a point of time into a vector, taking into account how different coordinates of time referring to the same date can affect the dynamics of a model.

\section{Introduction}

This work belongs to a series of papers (see [1-4]) which explore the notion of multitime in different branches of mathematical analysis, such as economics and physics. Since 1932, the adjective multitime, introduced by Paul Dirac, appears so as to cope with an environment in which there might be more than one dimension of time.

We make some considerations concerning the physical idea of the multitime notion: we recall that a coordinate space is an index numbering degrees of freedom, and the coordinate of time is the usual physical time in which a system evolves. This model is satisfactory, unless we turn our attention to relativistic problems. Moreover, some physical phenomena (and social sciences too) are observed in a twotime environment $t=\left(t^{1}, t^{2}\right)$, in which $t^{1}$ is the intrinsic time and $t^{2}$ is the observer time. In some real phenomena, there is no reason to prefer one coordinate to another. Following these assumptions, we refer to multitime as vector parameters of evolution in multitime geometric evolution and multitime optimal control problems.

The second relevant aspect to analyze is the multitime wave functions considered by Paul Dirac in 1932 via $m$-time evolution equations $i h\left(\partial \psi / \partial t^{\alpha}\right)=H_{\alpha} \psi$. The Dirac PDEs system is consistent (completely integrable) if and only if $\left[H_{\alpha}, H_{\beta}\right]=0$ for $\alpha \neq \beta$. The consistency condition is easy to achieve for noninteracting particles, while it turns out to be harder in the presence of interactions. All of these laws cannot be applied to relevant problems, if one aims at a plain covariant formulation of relativistic quantum mechanics.

Eventually, following the literature, we can divide the laws of evolution of physical theories into two branches: the singletime evolution laws (ODEs) and the multitime evolution laws (PDEs). It is clear that, in order to turn a single-time evolution into a multitime evolution, changing the ODEs into PDEs is enough by accepting that time $t$ is $C^{\infty}$ function of certain parameters; let us say that $t=t\left(s^{1}, \ldots, s^{m}\right)$.

Many authors studied the different applications of these notions: we recall some significative papers as [4-10] in which the PDEs constraints usually show significant challenges for optimization principles. The multivariable maximum principle was studied in the presence of PDE constraints (see [1]).

The multivariate recurrences are based on multiple sequences; they come from areas like analysis of algorithms, computational biology, information theory, queueing theory, filters theory, statistical physics, and so forth. We consider a lattice of points with positive integer coordinates in $\mathbb{R}^{n}$. A 
multivariate recurrence is a set of rules which transfer a point to another, together with initial conditions, which is capable of covering the hole lattice.

A multivariate recurrence relation is an equation that recursively defines a multivariate sequence, once one or more initial terms are given: each further term of the sequence is defined as a function of the preceding terms. Some defined recurrence relations can have very complex (chaotic) behaviors, and they are part of a field of mathematics known as nonlinear analysis.

We shall extend the Floquet theory which had been firstly utilized for periodic linear ODEs [11] and then extended to difference equations $[12-15]$. In $[3,16]$, the authors have extended this theory to the multitemporal first-order PDEs. In Floquet theory, it is necessary to find the associated monodromy matrix and its eigenvalues (called Floquet multipliers) in order to pass from a constant coefficient state to a periodic one. It can be crucial in a recurrence formulation (as noted in [14]) in which time-independent coefficients are unrealistic. An application of the Floquet theory can be found in [14]; here, we shall discuss a further possible extension. In this case, there is a generalization of the periodicity to a sheet of time rather than a point of time approach. In few words, it can be stated that, by a multitime approach, a point of time opens up to a vector of multiple times at that date (see Figure 1).

\section{The Model}

2.1. A Hint on the Samuelson-Hicks Model. The standard Samuelson-Hicks model has been made of two crucial components: $\gamma$, the propensity to consume, for which amount $C_{t}=\gamma Y_{t-1}$ is consumed, in which $Y_{t-1}$ is the income at $t-1$, and the rest is saved. The second component is that productive capital needs to aggregate production in fixed proportion $\alpha: K_{t}=\alpha Y_{t-1}$. Defining the investment as a change in capital stock $I_{t}=K_{t}-K_{t-1}$, then $I_{t}=\alpha\left(Y_{t-1}-Y_{t-2}\right)$, that is, the standard principle of acceleration. Furthermore, since $Y_{t}=C_{t}+I_{t}$, then a single recurrence equation in income is

$$
Y_{t}=(\alpha+\gamma) Y_{t-1}-\alpha Y_{t-2}
$$

In [17], Samuelson applied the accelerator to consumption only, while, in [18], Hicks described its application to all expenditures including floors to depreciation levels and ceilings to incomes growth.

The equations of the model encompass autonomous factors $\bar{C}$ (consumption independent from income) and $\bar{I}$ (investment not dependent on business cycles): $C_{t}=\gamma Y_{t-1}+\bar{C}$ and $I_{t}=\alpha\left(Y_{t-1}-Y_{t-2}\right)+\bar{I}$. Notice that these autonomous components are crucial for the determination of the cycle. The induced investment depends on the change in output (on the cycle), while the autonomous part depends on technological progress, innovation, or population growth. If the autonomous investment increases at a regular rate, then the economy is in a progressive equilibrium.

A problem may arise: autonomous components grow at a constant rate, and $\alpha$ and $\gamma$ do not depend upon the cycle

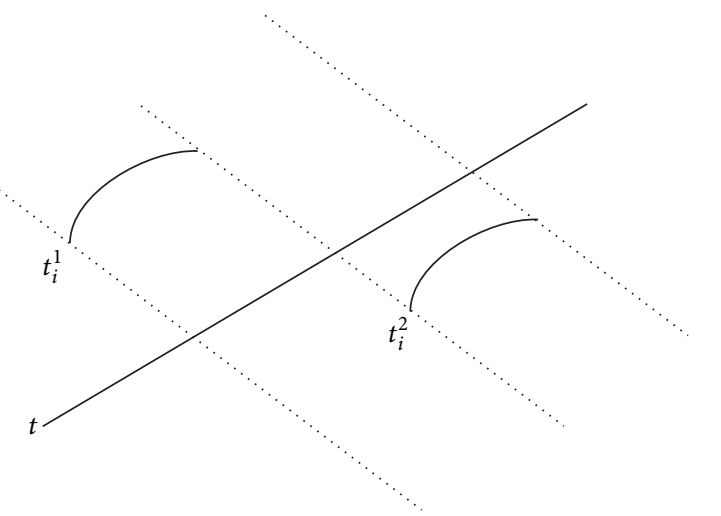

FIgURE 1: An example of multitime sheet.

and are assumed to be constant too. In [14], these constant factors become periodic and then they are floquetized in the sense of the following section (even if they, and we, detrended the model since growing autonomous investments have been removed). Moreover, we shall discuss a further generalization of the Floquet periodicity in which every period is decomposed into a vector of times rather than a simple point.

2.2. From Floquetizations to Multitime. Define element $t=$ $\left(t^{1}, \ldots, t^{m}\right) \in \mathbb{N}^{m}$ as a discrete multitime and function $x$ : $\mathbb{N}^{m} \rightarrow \mathbb{R}^{n}$ as a multivariate sequence. Moreover, denote $\mu(t)=$ $\min \left\{t^{1}, \ldots, t^{m}\right\}$ and $\mathbf{1}=(1, \ldots, 1) \in \mathbb{N}^{m}$. It seems intuitive to see the discrete multitime as a series of vectors in which the time is not seen as a simple straight line in $\mathbb{R}$ and every time as a point on that line; with the multitime approach, every point of time is turned into a vector. In general, in economics, a model is defined in either discrete or continuous time: in the former case, it is usual to say that a generic event happens at $t=1$ and spreads its effects at $t=2$ and so forth. For a multitime approach, an event does not come into being at $t=i \in \mathbb{N}$ but at $t=t^{i} \equiv\left(t_{1}^{i}, t_{2}^{i}, t_{3}^{i}, \ldots, t_{m}^{i}\right)$; it can be thought of as a sheet of time instead of a point (see Figure 1). An event that occurred on a sheet of time $t^{1}$ spreads its effects on the subsequent sheet of time $t^{2}$ and so on.

Periodic coefficients are a proper generalization of constant ones, since the former take into account the periodical characteristic. This generalization can be applied to the Samuelson-Hicks model with constant coefficient and try to understand if generalizing it to a periodic coefficient model may be of some interest. This approach is called floquetization, in particular for coefficient $h_{t}$

$$
h_{t+\theta}=z h_{t}
$$

then it can be defined as a floquetian of type $(\theta, z)$, in which when $z=1$, it is nothing more than $\theta$-periodicity. If $z \neq 1$, then floquetian coefficiencies are a straightforward generalization of the $\theta$-periodic ones. The discrete multitime 
periodic coefficient model is a further generalization to a multitime approach, where

$$
\begin{aligned}
& h(t+\theta)=A(t) h(t), \\
& h(t+\mathbf{1})=A(t) h(t) \quad \text { with } \theta \equiv \mathbf{1},
\end{aligned}
$$

where, in case of $m \geqslant 2, A: \mathbb{N}^{m} \rightarrow \mathscr{M}_{n}(\mathbb{R})$. In general, for any $T \in \mathbb{N}$, the form

$$
A(t+T \cdot \mathbf{1})=A(t)
$$

is called T-diagonal periodic.

2.3. Samuelson-Hicks Meets Multitime. The discrete multitime periodic coefficient Samuelson-Hicks model can be written as a first order diagonal recurrence system of the form of (4)

$$
\left(\begin{array}{c}
Y(t+\mathbf{1}) \\
C(t+\mathbf{1})
\end{array}\right)=\left(\begin{array}{cc}
\gamma(t)+\alpha(t) & -\frac{\alpha(t)}{\gamma(t-\mathbf{1})} \\
\gamma(t) & 0
\end{array}\right)\left(\begin{array}{l}
Y(t) \\
C(t)
\end{array}\right)
$$

that satisfies the following conditions:

$$
\begin{gathered}
\left.Y(t)\right|_{t^{\alpha}=0}=Y_{0 \alpha}\left(t^{1}, \ldots, \widehat{t^{\alpha}}, \ldots, t^{m}\right), \\
\left.C(t)\right|_{t^{\alpha}=0}=C_{0 \alpha}\left(t^{1}, \ldots, \widehat{t^{\alpha}}, \ldots, t^{m}\right), \\
\left.Y_{0 \alpha}\left(t^{1}, \ldots, \widehat{t^{\alpha}}, \ldots, t^{m}\right)\right|_{t^{\beta}=0} \\
\quad=\left.Y_{0 \beta}\left(t^{1}, \ldots, \widehat{t^{\beta}}, \ldots, t^{m}\right)\right|_{t^{\alpha}=0}, \\
\left.C_{0 \alpha}\left(t^{1}, \ldots, \widehat{t^{\alpha}}, \ldots, t^{m}\right)\right|_{t^{\beta}=0} \\
=\left.C_{0 \beta}\left(t^{1}, \ldots, \widehat{t^{\beta}}, \ldots, t^{m}\right)\right|_{t^{\alpha}=0} .
\end{gathered}
$$

The marginal propensity to consume $0<\gamma<1$; moreover, $\alpha$ can be defined as decelerator if $0<\alpha<1$, keeper if $\alpha=1$, and accelerator if $\alpha>1$. National income $Y$ and its endogenous variable $C$ (consumption) are assumed as strictly positive multitime sequences $Y(t)$ and $C(t)$. Notice that, in the original model, $\gamma$ and $\alpha$ are constant factors. In [14], they have been used as $(\theta, z)$-periodic parameters $\gamma_{t}$ and $\alpha_{t}$ as the autonomous consumption $\bar{C}$ turned into $C_{t}$. This periodicity has been extended to $(\theta, A)$-periodic parameters $\gamma(t), \alpha(t)$, $Y(t), C(t)$.

The model may be written as the second order homogeneous diagonal recurrence equation:

$$
\begin{gathered}
Y(t+\mathbf{2})-(\gamma(t+\mathbf{1})+\alpha(t+\mathbf{1})) Y(t+\mathbf{1}) \\
+\alpha(t+\mathbf{1}) Y(t)=0, \quad t \in \mathbb{N}^{m},
\end{gathered}
$$

satisfying the following conditions:

$$
\begin{aligned}
& Y(t+2)-(\gamma+\alpha) Y(t+1)+\alpha Y(t)=0, \quad t \in \mathbb{N}^{m}, \\
& \left.Y(t)\right|_{t^{\alpha}=0}=Y_{0 \alpha}\left(t^{1}, \ldots, \widehat{t^{\alpha}}, \ldots, t^{m}\right) \\
& \left.Y(t)\right|_{t^{\alpha}=1}=Y_{1 \alpha}\left(t^{1}, \ldots, \widehat{t^{\alpha}}, \ldots, t^{m}\right) \\
& \left.Y_{0 \alpha}\left(t^{1}, \ldots, \widehat{t^{\alpha}}, \ldots, t^{m}\right)\right|_{t^{\beta}=0} \\
& \quad=\left.Y_{0 \beta}\left(t^{1}, \ldots, \widehat{t^{\beta}}, \ldots, t^{m}\right)\right|_{t^{\alpha}=0}, \\
& \left.Y_{1 \alpha}\left(t^{1}, \ldots, \widehat{t^{\alpha}}, \ldots, t^{m}\right)\right|_{t^{\beta}=1} \\
& \quad=\left.Y_{1 \beta}\left(t^{1}, \ldots, \widehat{t^{\beta}}, \ldots, t^{m}\right)\right|_{t^{\alpha}=1}, \\
& \left.Y_{0 \alpha}\left(t^{1}, \ldots, \widehat{t^{\alpha}}, \ldots, t^{m}\right)\right|_{t^{\beta}=1} \\
& \quad=\left.Y_{1 \beta}\left(t^{1}, \ldots, \widehat{t^{\beta}}, \ldots, t^{m}\right)\right|_{t^{\alpha}=0} .
\end{aligned}
$$

A prominent role is played by the so-called Floquet multiplier of (8), that is, first reconverting (8) into an equivalent diagonal recurrence system:

$$
\begin{aligned}
& \left(\begin{array}{c}
Y(t+\mathbf{1}) \\
Z(t+\mathbf{1})
\end{array}\right) \\
& \quad=\underbrace{\left(\begin{array}{cc}
0 & 1 \\
-\alpha(t+\mathbf{1}) & \gamma(t+\mathbf{1})+\alpha(t+\mathbf{1})
\end{array}\right)}_{=A(t+\mathbf{1})}\left(\begin{array}{c}
Y(t) \\
Z(t)
\end{array}\right),
\end{aligned}
$$

where $A(t+\mathbf{1})$ is $T$-periodic matrix. Then, find the Floquet multiplier, which is an eigenvalue of the matrix:

$$
C(t)=\prod_{k=0}^{T-1} A(t+(T-k-\mu(t) \cdot \mathbf{1})),
$$

that is called the monodromy matrix. It is a $2 \times 2$ matrix with determinant

$$
\begin{aligned}
\operatorname{det} C(t)= & \alpha(t+(T-\mu(t)) \cdot \mathbf{1}) \times \cdots \\
& \times \alpha(t+(1-\mu(t)) \cdot \mathbf{1}) .
\end{aligned}
$$

Thus, the multipliers are the roots of the quadratic equation:

$$
z^{2}-z(\operatorname{Tr} C)+\operatorname{det} C=0
$$

and these multipliers will depend on $t^{1}-\mu(t), \ldots, t^{m}-\mu(t)$.

\section{Discussion and Conclusions}

The multiplier-accelerator model shows how cycles can occur in economics. The Floquet theory can be used to 
generalize the constant coefficient framework of the standard Samuelson-Hicks model. By floquetizing this model, the behavior of the system around their constant value can be studied and thus it is possible to study the periodic cycle. This can happen by the use of the monodromy matrix which is the link between the coefficients and their periodic generalization. These kind of matrices study the behavior of a dynamic system around a singularity, in this case a constant coefficient.

It can be stated that each motion of national income $Y$ is bounded in (8) if and only if this motion is bounded and $\alpha \leqslant 1$ in (12). For $\alpha>1$, it is unbounded and, moreover, each motion of $Y$ vanishes if $\alpha<1$.

The floquetized Samuelson-Hicks model has been further generalized by taking into account a discrete multitime model generalizing the point of time to a sheet of time, as mentioned above. By a sheet of time, the dynamic system may be decomposed into different summable time vectors in which it is possible to study the effect of each coefficient separately. It can be possible to explode the model into different current times, like those paintings from the analytical cubism. It is important to apply the model so as to study recursively the effect of lagged coefficients to the subsequent ones, but the new effect which may become crucial is that in each sheet one can observe a decomposed model: on every time encompassed by a sheet, one can see how modifications of a coefficient affect the coefficients in the same current time sheet and so how this vector affects the model in the other sheet by understanding that model thoroughly.

\section{Competing Interests}

The authors declare that there are no competing interests regarding the publication of this paper.

\section{Acknowledgments}

The authors thank Massimiliano Ferrara for his invaluable advice and guidance. This research was partially supported by a grant of the Department of Law and Economics of the University Mediterranea of Reggio Calabria.

\section{References}

[1] C. Udrişte, "Multitime controllability, observability and bangbang principle," Journal of Optimization Theory and Applications, vol. 139, no. 1, pp. 141-157, 2008.

[2] C. Udriste and M. Ferrara, "Multitime models of optimal growth," WSEAS Transactions on Mathematics, vol. 7, no. 1, pp. 51-55, 2008.

[3] C. Udrişte, M. Ferrara, and D. Opris, Economic Geometric Dynamics, vol. 6 of Mono-Graphs and Textbooks, Geometry Balkan Press, Bucharest, Romania, 2004.

[4] C. Udriște, M. Ferrara, D. Zugrăvescu, and F. Munteanu, "Controllability of a nonholonomic macroeconomic system," Journal of Optimization Theory and Applications, vol. 154, no. 3, pp. 1036-1054, 2012.
[5] V. Barbu, I. Lasiecka, D. Tiba, and C. Varsan, "Analysis and optimization of differential systems, IFIP TC7/WG7.2," in Proceedings of the International Working Conference on Analysis and Optimization of Differential Systems, Kluwer Academic, Constanta, Romania, September 2002.

[6] G. Barles and A. Tourin, "Commutation properties of semigroups for first-order Hamilton-Jacobi equations and application to multi-time equations," Indiana University Mathematics Journal, vol. 50, no. 4, pp. 1523-1544, 2001.

[7] A. M. Bayen, R. L. Raffard, and C. J. Tomlin, "Adjointbased constrained control of Eulerian transportation networks: application to air traffic control," in Proceedings of the American Control Conference (AAC '04), pp. 5539-5545, Boston, Mass, USA, July 2004.

[8] F. Cardin and C. Viterbo, "Commuting Hamiltonians and Hamilton-Jacobi multi-time equations," Duke Mathematical Journal, vol. 144, no. 2, pp. 235-284, 2008.

[9] M. Motta and F. Rampazzo, "Nonsmooth multi-time HamiltonJacobi systems," Indiana University Mathematics Journal, vol. 55, no. 5, pp. 1573-1614, 2006.

[10] S. Pickenhain and M. Wagner, "Piecewise continuous controls in Dieudonné-Rashevsky type problems," Journal of Optimization Theory and Applications, vol. 127, no. 1, pp. 145-163, 2005.

[11] G. Floquet, "Sur les èquations diffèrentielles linèaires á coefficients pèriodiques," Annales Scientifiques de l'École Normale Supérieure, vol. 12, pp. 47-88, 1883.

[12] P. A. Kuchment, Floquet Theory for Partial Differential Equations, Birkhauser, 1993.

[13] N. G. Markley, Principles of Differential Equations, Pure and Applied Mathematics (New York), John Wiley \& Sons, New York, NY, USA, 2004.

[14] P. V. Mouche and W. Heijman, Floquet Theory and Economic Dynamics (Extended version), Wageningen Economic Papers, Wageningen, The Netherlands, 1996.

[15] V. A. Yakubovich and V. M. Starzhinskii, Linear Differential Equations with Periodic Coefficients, vol. 1, John Wiley \& Sons, New York, NY, USA, 1975.

[16] C. Udrişte, "Multitime floquet theory," Atti della Accademia Peloritana dei Pericolanti-Classe di Scienze Fisiche, Matematiche e Naturali, vol. 91, no. 2, article A5, 2013.

[17] P. A. Samuelson, "Interactions between the multiplier analysis and the principle of acceleration," Review of Economic Statistics, vol. 21, no. 2, pp. 75-78, 1939.

[18] J. R. Hicks, A Contribution to the Theory of the Trade Cycle, Oxford University Press, Oxford, UK, 1950. 


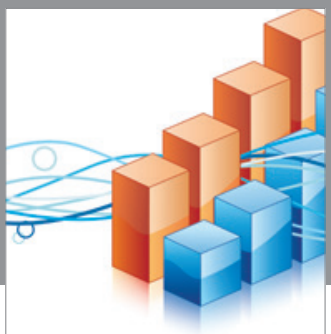

Advances in

Operations Research

vatem alat4

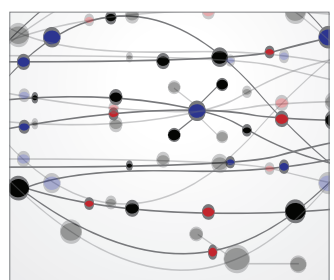

\section{The Scientific} World Journal
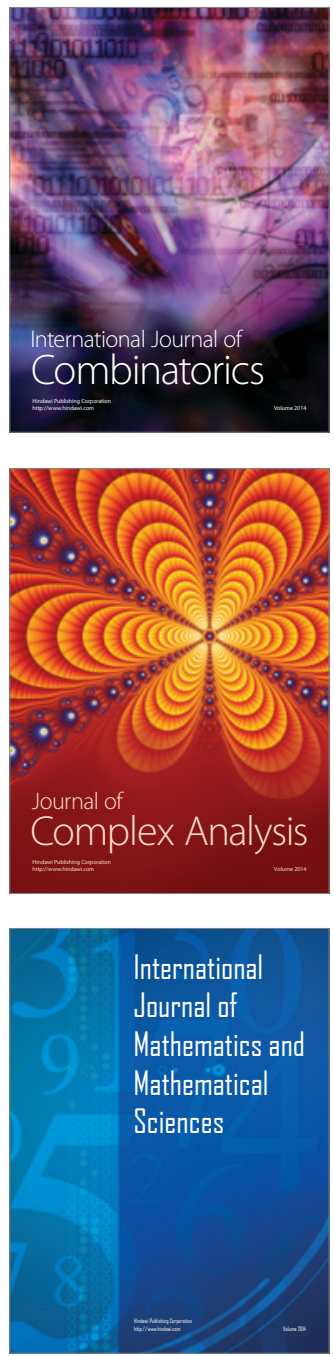
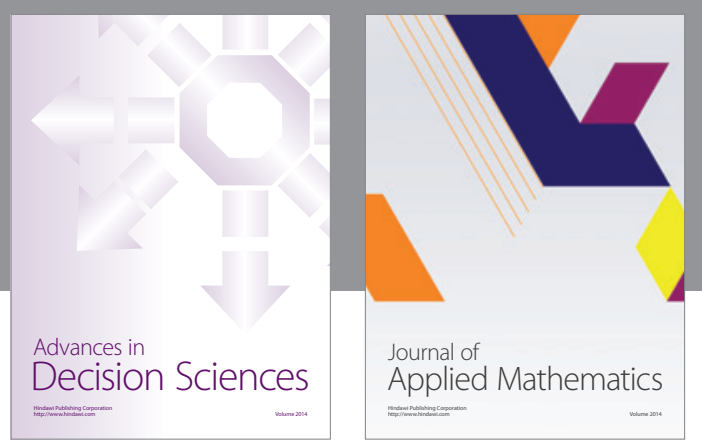

Algebra

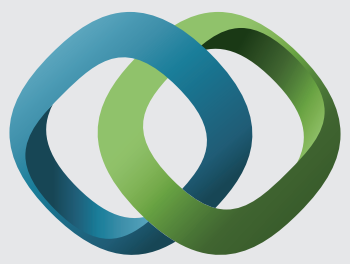

\section{Hindawi}

Submit your manuscripts at

http://www.hindawi.com
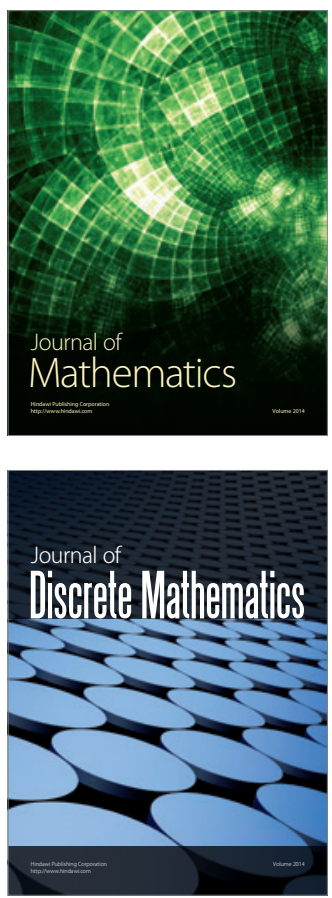

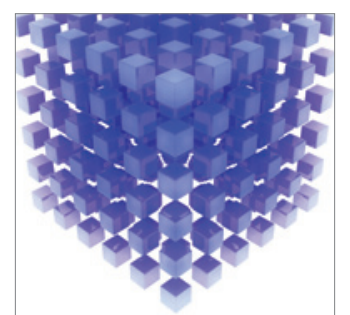

Mathematical Problems in Engineering
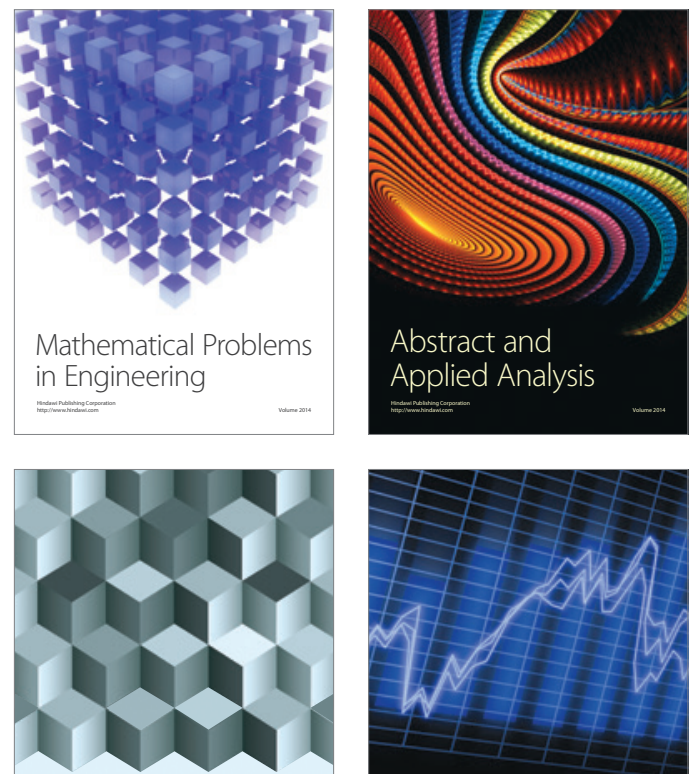

Journal of

Function Spaces

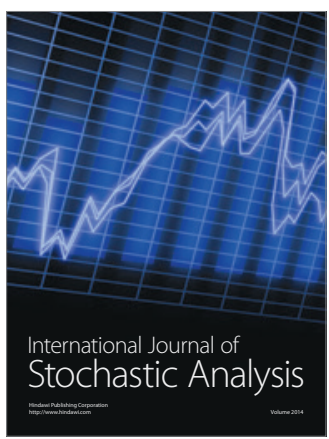

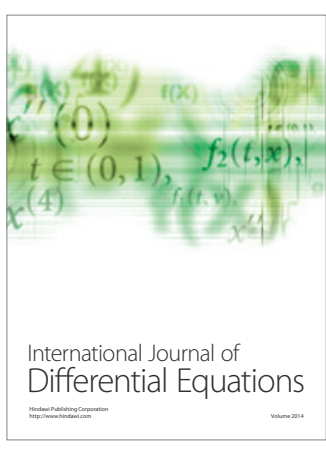
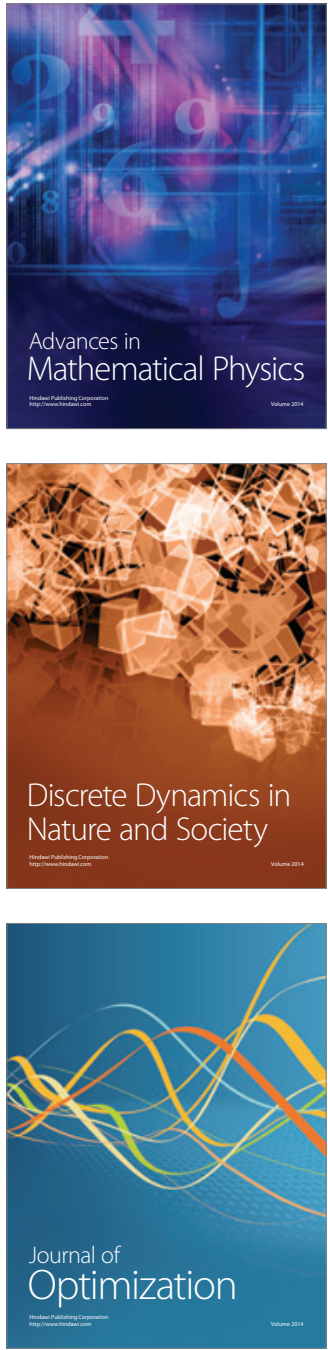